\title{
The present and future of cough counting tools
}

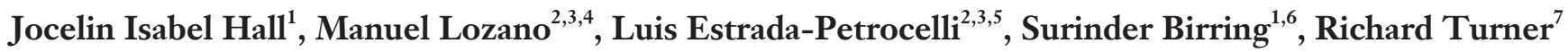 \\ ${ }^{1}$ Centre for Human and Applied Physiological Sciences, King's College London, London, UK; ${ }^{2}$ Institute for Bioengineering of Catalonia (IBEC), \\ The Barcelona Institute of Science and Technology (BIST), Barcelona, Spain; ${ }^{3}$ Biomedical Research Networking Centre in Bioengineering, \\ Biomaterials and Nanomedicine (CIBER-BBN), Barcelona, Spain; ${ }^{4}$ Department of Automatic Control (ESAII), Universitat Politècnica de \\ Catalunya (UPC)-Barcelona Tech, Barcelona, Spain; ${ }^{5}$ Facultad de Ingeniería, Universidad Latina de Panamá, Panama City, Panama; ${ }^{6}$ Department \\ of Respiratory Medicine, King's College Hospital NHS Foundation Trust, London, UK; ${ }^{7}$ Department of Respiratory Medicine, Charing Cross \\ Hospital, Imperial College Healthcare NHS Trust, London, UK \\ Contributions: (I) Conception and design: All authors; (II) Administrative support: None; (III) Provision of study materials or patients: None; (IV) \\ Collection and assembly of data: None; (V) Data analysis and interpretation: None; (VI) Manuscript writing: All authors; (VII) Final approval of \\ manuscript: All authors. \\ Correspondence to: Dr. Richard Turner. Department of Respiratory Medicine, Charing Cross Hospital, Imperial College Healthcare NHS Trust, \\ London, UK. Email: richard.turner17@nhs.net.
}

\begin{abstract}
The widespread use of cough counting tools has, to date, been limited by a reliance on human input to determine cough frequency. However, over the last two decades advances in digital technology and audio capture have reduced this dependence. As a result, cough frequency is increasingly recognised as a measurable parameter of respiratory disease. Cough frequency is now the gold standard primary endpoint for trials of new treatments for chronic cough, has been investigated as a marker of infectiousness in tuberculosis (TB), and used to demonstrate recovery in exacerbations of chronic obstructive pulmonary disease (COPD). This review discusses the principles of automatic cough detection and summarises key currently and recently used cough counting technology in clinical research. It additionally makes some predictions on future directions in the field based on recent developments. It seems likely that newer approaches to signal processing, the adoption of techniques from automatic speech recognition, and the widespread ownership of mobile devices will help drive forward the development of real-time fully automated ambulatory cough frequency monitoring over the coming years. These changes should allow cough counting systems to transition from their current status as a niche research tool in chronic cough to a much more widely applicable method for assessing, investigating and understanding respiratory disease.
\end{abstract}

Keywords: Cough; cough monitor; cough frequency

Submitted Mar 24, 2020. Accepted for publication Aug 19, 2020.

doi: $10.21037 /$ jtd-2020-icc-003

View this article at: http://dx.doi.org/10.21037/jtd-2020-icc-003

\section{Introduction}

Although advances over the last 10-20 years have progressed understanding of the mechanisms and pathophysiology of cough, and of the impact of cough on patients, much remains poorly understood (1). These developments have occurred along with, and have been strongly supported by, tools for measuring cough (2).

As has been reviewed elsewhere (2-6), cough can be measured in a number of ways. Clinically, subjective measures are the most routinely employed, either crudely through qualitative questioning, or more formally through quantitative assessments including the Leicester Cough Questionnaire (7), cough severity visual analogue scale (VAS) (8), cough severity diary (CSD) (9) and the coughspecific quality of life questionnaire (CQLQ) (10). These subjective assessments are straightforward and important, allowing a valuable insight into the impact of the cough on the individual (11). However, by nature of their subjectivity, such tools are a proxy measure only, of a 
A

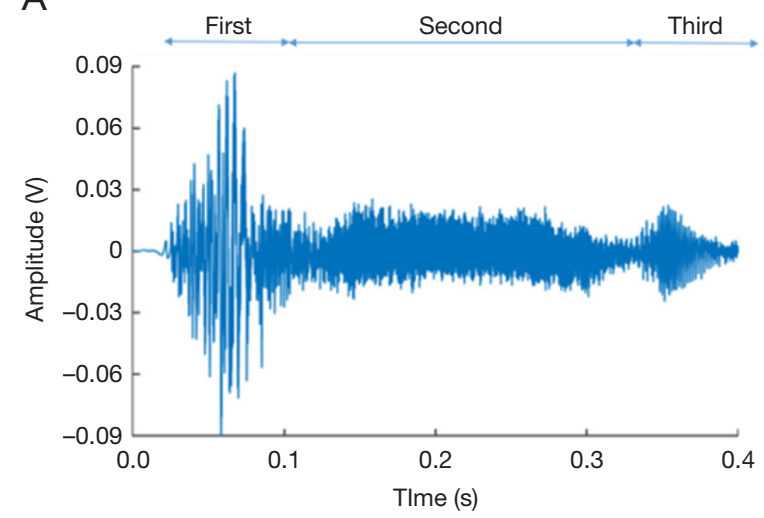

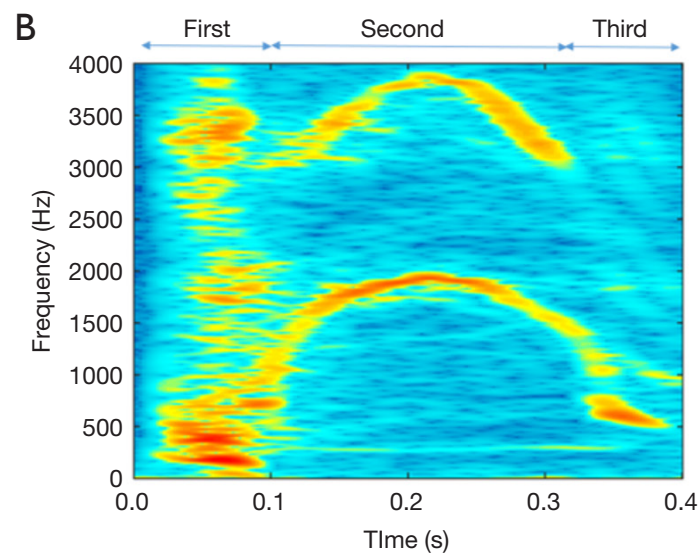

Figure 1 The component phases of the cough sound: opening of the vocal cords (first phase), air flow through the open larynx (second phase), and re-apposition of the cords (third, voiced, phase-not always present). Shown as changes in sound amplitude (A) and frequency (B).

patient's perception of actual coughing events, rather than a direct assessment of cough itself. Subjective and objective measures show only moderate correlation at best (12).

Objective measurement aims to offer an impartial quantification of the physiological and pathological phenomenon of cough. Although it is possible to measure different physical characteristics, including force or intensity $(13,14)$, and acoustic properties of cough $(15)$, the most widely measured objective variable for assessing cough is its frequency.

There are several reasons why cough frequency measurements might be useful. Chronic cough is a common disorder, affecting approximately $10 \%$ of the population (16), and is associated with significant morbidity $(17,18)$. Cough counts in individual patients are an objective marker of cough severity, variation over time may suggest triggers or aetiologies, and repeated measures following treatments can assess their efficacy. With the wider use of cough monitoring in research, objective cough counts are now becoming primary endpoints in clinical trials of anti-tussive therapies $(19,20)$.

Cough frequency measurement may also be useful for monitoring treatment of other respiratory diseases (21), assessing infectiousness in tuberculosis (TB) (22), detecting early signs of exacerbations of chronic respiratory disease (23), and possibly for screening for the early stages of potentially treatable diseases including lung cancer and TB.

For the time being, at least to our knowledge, cough frequency monitoring has not been incorporated into routine clinical practice and remains a research tool. This review will provide an up-to-date overview of cough counting tools currently and recently used in research, discuss technological aspects, and speculate on possible future developments in the field.

Due to the scope of this review and the constantlyevolving nature of the field, it does not aim to be comprehensive, but rather to offer the reader insights by focusing on key principles, and on technology and devices which have led to significant advances in the understanding and management of cough.

\section{Defining cough}

Cough is characterised by three stages. During the first, inspiratory stage, air is drawn into the lungs. This is followed by the compressive stage characterised by forced expiratory effort against the closed glottis. Finally, during the expulsive stage there is opening of the glottis and rapid outflow of air. This sudden release of rapid and turbulent expiratory airflow is responsible for the characteristic sound of a cough which essentially defines it $(24,25)$. This third stage itself usually has three component phases which comprise the cough sound, as described in Figure 1 and below.

Coughs often occur close together in clusters, which may be described as epochs, bouts, peals or attacks. During these, the initial inspiratory phase is followed by a series of further compressive phases associated with glottal closure, sometimes with additional inspirations (24). A bout or epoch is defined as a cluster of two or more cough sounds, separated from the next by an interval of no more than 2 seconds (24). 
Different units have been proposed for cough counting, including individual cough sounds, cough bouts, and time spent coughing (26). No measure is demonstrably superior, but numbers of cough sounds and time spent coughing are very closely correlated (27). Individual cough sounds, either occurring in isolation or as part of a cluster, are probably therefore the most intuitive basic units of cough $(25,28)$.

\section{Establishing a reference standard for counting coughs}

The assumed gold standard method for counting coughs, against which potential automated cough counters are evaluated, is the 'manual' counting of cough sounds from near-patient audio recordings. Within pairs, cough counts performed by experienced cough researchers are reported to be highly consistent $(29,30)$. One study though has specifically set out to test the validity of cough counting by ear amongst a larger group (28).

Fifteen doctors untrained in counting coughs were asked to individually listen to audio recordings, lasting around 15 minutes and containing coughs from patients with respiratory diseases. There was very close inter- and intra-observer agreement of the reported cough counts. The recordings contained a mixture of lone coughs and coughing bouts, and no specific instructions were given as to how coughs should be counted. The consistently reported values for cough frequency corresponded to the total numbers of individual cough sounds, providing further good justification not only for counting by ear as the reference standard for determining cough frequency, but also for using discrete cough sounds as the basic unit for cough counting (28).

\section{A brief history of cough frequency monitors}

Attempts at quantifying cough frequency over prolonged periods began in the 1950s (31). In 1964 Woolf and Rosenberg were able to demonstrate a reduction in cough frequency with anti-tussive therapy by counting coughs recorded from a microphone above the head of the bed connected through to a tape recorder (32). Recording was triggered by sound from the microphone and continued to record for 5 seconds after the sound had stopped, allowing it to record up to 24 hours of observations on 2 hours of tape. Manual counts of the audio recordings were then undertaken by the investigators. A similar system was developed by Loudon and colleagues in the 1960s
$(33,34)$. Again, due to limitations of technology at the time, equipment was bulky, and patients confined to a single room for the duration of monitoring, which in this case was up to 10 hours. The final recordings, also analysed by ear, similarly represented only certain portions of the full recording period, during which sounds meeting pre-specified amplitude and frequency criteria had triggered the audio capture apparatus. This inability to record for the full duration of the monitoring period presumably led to the omission of a proportion of coughing events with both systems.

In 1988 Salmi et al. made the first attempt at the automation of cough frequency monitoring (35). The researchers' apparatus recorded cough sounds via a microphone and body movements with a static chargesensitive mattress. Coughs had to breach specified acoustic and movement thresholds to be automatically identified. The method was evaluated by comparison to cough counts from a researcher simultaneously observing the patient in real time during the recording period. In 7 patients, the machine detected 809 cough events, of which 794 were true positives, showing a sensitivity of $99.0 \%$ and specificity of $98.1 \%$. The method was, however, extremely restrictive to subjects. They were required to remain sitting or lying in bed isolated in a single hospital room, told to avoid sudden movements and loud noises, and to remain facing away from the pillow. Presumably in part because of this, the system does not seem to have been developed any further.

The 1990s and early 2000s saw advances in technology which enabled the development of ambulatory devices (36). MP3 recording, digital storage, miniaturisation of microphones, and developments in battery technology facilitated the ability to record and capture high quality data continuously over prolonged periods (37).

Processing the recorded information however remained the main limiting step owing to a reliance on manual assessment. Counting coughs by ear from prolonged audio sequences is not only laborious and time-consuming, but auditory fatigue may lead to miscounting and errors; ideally a subset of recordings should be counted twice, by more than one observer, to determine consistency and quality control. Any steps towards full automation of cough counting would therefore be highly attractive.

\section{Principles of automatic cough detection}

Most previous studies focusing on cough detection have used a conventional approach to audio signal processing (Figure 2), applying techniques used in automatic speech 


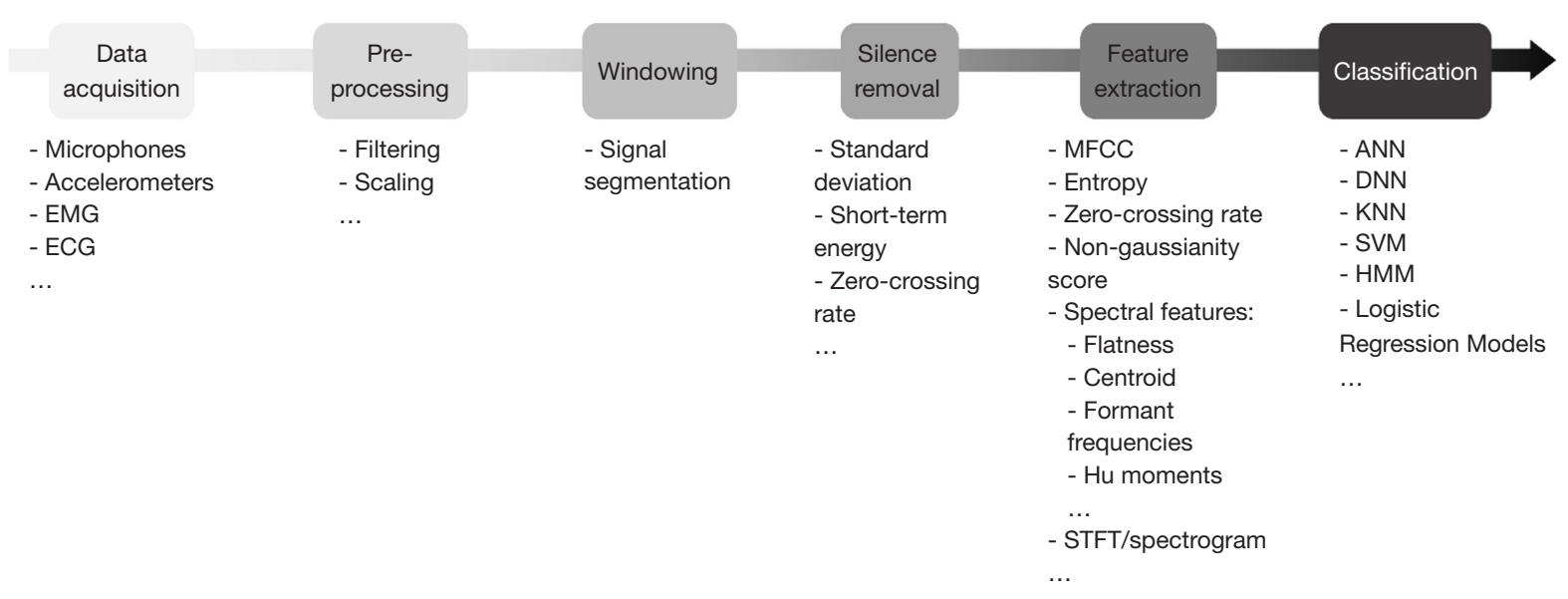

Figure 2 Scheme of analysis for automatic cough detection. EMG, electromyography; ECG, electrocardiography; MFCC, Mel-frequency cepstral coefficients; STFT, short-time Fourier transform; ANN, artificial neural network; DNN, deep neural network; KNN, K-nearest neighbours; SVM, support vector machines; HMM, hidden Markov models.

recognition. The core of this strategy involves three steps: silence removal, feature extraction and classification. However, different methods have been proposed for these steps, and there is currently no standardised methodology for automatic cough detection.

\section{Cough signal capture}

The most studied signal for cough evaluation is sound, acquired by means of microphones. Other investigated modalities include: electromyography (EMG), electrocardiography (ECG), nasal thermocouple sensors, effort belts, and accelerometry (21), individually or together (38-42). Microphones can be classified as contact, and noncontact $(43,44)$. Non-contact microphones are either worn on the outer clothing or placed in the subject's vicinity and detect fluctuations in air pressure which are converted into electrical signals by transduction. Contact microphones are attached to the skin surface and use piezoelectric transducers to sense audio vibrations through direct physical contact. Although less sensitive to ambient noise than noncontact microphones, their high sensitivity is prone to noise from movement artefact (45).

An array or combination of microphones may enhance the spatial filtering selectivity of recordings and improve cough discrimination, particularly in noisy environments $(46,47)$.

A cough sound can be described as a non-stationary signal and, as discussed, separated into three component phases: the explosive phase, intermediate phase, and voiced phase (Figure 1). A cough lasts on average $350 \mathrm{~ms}$, with a first peak of mean frequency c. $400 \mathrm{~Hz}$, a secondary peak of highest continuous frequency c. 4,000 Hz (48), and frequency components of sound spread up to $20 \mathrm{kHz}(49)$. To properly characterize cough events the complete capture of all details from the audio signal is necessary. A range of sampling frequencies for the acquisition of the audio signal has been suggested, from $8 \mathrm{kHz}$ (50) to $48 \mathrm{kHz}$ (51). The frequency response of the microphone must be within the sound frequency range of the coughing events. In addition, the selection of a sampling rate must consider the highest frequency component of the cough sounds; as stated by the Nyquist sampling theorem, components above half the sampling rate must be filtered out to avoid aliasing (52). The sampling rate will impact on the volume of data acquired; lower rates reduce hardware and data storage requirements, as well as time needed to carry out automated analysis, which are highly relevant for the development of practical applications.

\section{Windowing and silence removal}

Cough sound signals are commonly split into signal segments using a moving window, so that all subsequent analyses are performed on each segment (53). Prior to extracting information from the cough signal, preprocessing of the audio data is necessary with the aim of removing sources of noise that obscure its evaluation. In general, cough events are segmented and separated from other noise sources, a method known as silence removal (54). 
Silence removal methods can be performed manually or using automated methods such as standard deviation, shortterm energy, and zero-crossing (54). Standard deviation is the measure of dispersion of signal segments. A lower standard deviation refers to segments of low activity while higher standard deviation indicates segments with potential cough activity. Short-term energy represents the signal power. A low signal power is related to silence periods while a high signal power indicates a potential cough activity. Zero-crossing rate represents the number of times the signal crosses to zero. The higher the zero-crossing, the greater the amount of noise.

As suggested by Cohen-McFarlane et al., one step forward to improve silence removal and segmentation methods could be to implement an adaptive approach that improves the ability of separating background noise from audio activity (54). Nonetheless, these silence removal methods have limitations and work best in quiet ambient conditions, but lead to poorer segmentation in the case of low signal-to-background levels.

\section{Feature extraction}

Mel-frequency cepstral coefficients (MFCC) and their variations have been the most widely-used features for cough detection (41,55-65). MFCC, which are widely used in automatic speech recognition, represent the envelope of the short-term power spectrum of a sound signal. Spectral shape features may also help to differentiate cough from other sounds, particularly speech; compared to speech, cough sounds have a closer similarity to noise, and therefore have a wider spectrum.

Cough detection algorithms to date have included several distinct spectral features. Spectral flatness $(41,50,66)$ represents how flat the spectrum of a signal is or how similar to noise a sound is; spectral centroid $(41,50,66,67)$ concerns the weighted mean of the spectrum, usually higher in cough sounds than in other sounds; formant frequencies $(50,56,59,64)$ are spectral peaks at the resonant frequencies associated with cough generation; and spectral kurtosis $(59,64,67-69)$ measures how peaked the spectrum is.

Cough sounds are more noise-like than other sounds, and therefore are more complex. Accordingly, different measures of complexity of cough sound signals have also been proposed for cough detection, such as zerocrossing rate $(41,45,54,56,57,59,64,66)$ or different entropy measurements $(56,57,67,68)$.
Other features have been used for cough detection. These may include: the non-Gaussianity score $(56,57,59,64,70)$, quantifying the deviation of a signal from a Gaussian model, the value of which is typically high in cough sounds; log-energy $(59,61,64)$, relating to the amplitude of cough signals; and Hu moments, a technique of weighted averaging widely used in image processing and recently proposed in the signal processing field for speech emotion recognition $(67,71,72)$. Usually, these and other features are combined to form the input data set for classification. However, there is no consensus standard for the optimum set of acoustic features for cough detection.

\section{Classification}

Classification techniques aim to categorise sounds into cough and non-cough events. Artificial neural networks (ANN) are algorithms that attempt to simulate the behaviour of the human brain, and have been applied to attempt to differentiate between cough and non-cough events (56), cough segments and swallow signals, rest states and different non-cough artefacts (73), and to differentiate between cough, speech and noise (74). Recently, with advances in deep learning techniques, new approaches to cough detection have been proposed $(55,62,75)$. These approaches do not require feature extraction prior to classification, but the short-time Fourier transform or spectrogram of the cough sound signal is passed directly to a deep neural network within which feature extraction is performed automatically, thus facilitating the processing of cough sound signals.

The hidden Markov model (HMM) is a statistical technique successfully used for speech recognition. HMMs represent the spectral properties of a time-varying pattern of cough and have been employed for the automatic detection of cough events in ambulatory patients with respiratory diseases (65). In addition, hybrid models combining ANN and HMM (74), and DNN along with HMM (62) have been proposed to enhance the performance of cough detection by taking into account temporal variations in the cough signal.

Logistic regression classification is a kind of predictive analysis algorithm that assigns observations to a discrete (e.g., binary) set of classes. This technique has been used for the diagnosis of pertussis by evaluating cough and whoop sounds (76), and to separate cough and non-cough events $(50,77)$. Other interesting applications make use of support vector machine, an algorithm that it is suitable for the 
analysis of small data sets and that has being employed to improve automatic a croup diagnosis system (78).

Linear discriminant analysis (LDA) is a technique used for dimensionality reduction increasing separation between classes allowing classification tasks. LDA has been employed to obtain the best probable separation between cough and non-cough events (56).

The accelerated progress being made in the field of classification algorithms should allow more accurate and efficient classification between cough and non-cough events. The associated reduction in the computational cost could facilitate implementation in ambulatory scenarios, for example with incorporation into widely-available mobile devices as discussed below.

A major limitation when comparing different methods for automatic cough detection is that the datasets used in different studies are not the same, with main differences in type and position of microphones, recording conditions, study subjects and types of non-cough sounds included. Since these aspects influence the performance evaluation of methods, it is difficult to directly compare different approaches to automatic cough detection.

\section{Modern cough monitors}

The ideal ambulatory cough monitoring system would be easily portable, compact, minimally-intrusive to the patient, and probably operate over no less than a 24hour period to take account of diurnal variations in cough frequency (79). Such a device should reliably and consistently detect all coughs and distinguish them from all non-cough sounds, other respiratory movements, and ambient noise (i.e., high sensitivity and specificity), operate in a patient's own environment, function across a range of subjects, types of cough sound, in health, and in different diseases, and provide a fully automated analysis of the collected data. Ideally, cough monitors should also work in noisy environments and not mistake the coughs of other individuals for those of the subject of interest.

As discussed, the miniaturisation of electronic devices and the digitalisation of recording technology has led to wearable cough monitors capable of recording continuously for 24 hours or longer during daily activities. Despite various recent attempts at developing automated or semiautomated cough frequency monitors, some of which are described below, only two systems have been widely adopted in cough research: the Leicester Cough Monitor (LCM) and the VitaloJAK ${ }^{\mathrm{TM}}$ (37).

\section{The dextromethorphan trials}

In 2001 Pavesi et al. published a meta-analysis of 6 trials investigating the antitussive effect of dextromethorphan. Amongst 710 patients, cough reduction was demonstrated using cough frequency data collected by a portable computerised cough acquisition and analysis system, in the patient's own environment. The device consisted of a contact microphone, attached to the suprasternal notch, detecting audio and vibration signals. Data was collected by a frequency modulation transmitter, worn in a belt pouch, and sent wirelessly to the hardware within the patient's home. Subjects could move freely within a 100 -metre radius of the computer collecting the data. The system employed fully computerised acquisition of data but relied on manual counting of audio and visual displays.

Three hours of continuous cough recording was undertaken after treatment was initiated in each subject. Cough bouts, components, effort, intensity and latency were all measured. The antitussive effect of a single dose of dextromethorphan was demonstrated, and consistent results achieved by the cough counting system, showing for the first time the feasibility of portable cough monitors to evaluate treatment. Additionally the study showed cough frequency rather than intensity was a more responsive measure (80).

\section{The Lifeshirt ${ }^{\circledR}$}

The Lifeshirt ${ }^{\circledR}$ system was a multimodality automated cough counting tool comprising respiratory inductance plethysmography (RIP) for the non-invasive measurement of ventilatory variables, an accelerometer, single channel ECG, and a unidirectional throat microphone. The device was evaluated in one study of eight subjects with chronic obstructive pulmonary disease (COPD) against manual counts from video surveillance. The sensitivity and specificity of the device for counting coughs was reported as $78.1 \%$ and $99.6 \%$, respectively (81). There is no other published validation data; the company developing the product ceased trading and any further work looks unlikely. Nevertheless, this technology has influenced other products which are discussed below.

\section{The RBC-7, Logan Sinclair LR100 and LR102}

The RBC-7 recorded surface EMG and audio cough signals with a capacity for over 48 hours of data to an ambulatory 
device. Additional data from ECG and accelerometer supplied information on the subject's activity. Validation included data from 20 subjects and was compared to manual counting from tape recordings. No significant difference in the number of single coughs recorded by each system was detected (correlation coefficient $=0.996)(82)$. However, the system was not automated and therefore required full manual counts.

To overcome this operator dependence, modification with preset algorithms for data analysis was applied and the device re-named the LR100. It was validated in 14 children during exacerbations of cystic fibrosis (83). Further work in 2003 showed the feasibility of objective cough monitoring in younger infants and children. The system was shown to be well-tolerated and had a mean sensitivity of $81 \%$ for detecting coughs in comparison to human counts of video recordings (84).

The final update was to the LR102. This comprised 3 EMG sensors and a contact sound transducer. The EMG electrodes were placed across the chest: in the sixth right intercostal space, the left mid-clavicular region, and the epigastrium. The sound sensor was placed in the second left intercostal space. Data analysis was fully automated and off-line. Validation examined data collected from 10 adult patients with chronic cough. Cough frequency recorded by the LR102 and manual counting of video recordings were well correlated; $r=0.87$ for number of cough episodes/hour and $\mathrm{r}=0.89$ for number of single coughs/hour. However, the LR102 overestimated cough frequency. The mean difference between the meter and manual counts was 3.8 for cough episodes per hour $(\mathrm{P}=0.04)$ and 12.5 for single coughs per hour $(\mathrm{P}<0.01)$. This overestimation was due in large part difficulties of the automated system distinguishing between cough episodes and other noise. The shortcomings of the device have not been addressed and there have been no attempts to further develop it (85).

\section{Pulmotrack-CC $C^{T M}$}

The fully automated Pulmotrack-CC ${ }^{\mathrm{TM}}$ consisted of two contact microphones and a pneumogram belt. In a validation study by the developers it recorded tracheal and chest wall sounds, ambient sounds and chest wall motion in 12 healthy volunteers coughing voluntarily over short periods in 5 different positions: lying supine, sitting, sitting with high level ambient noise, walking and climbing stairs for short time periods ( 5 minutes/position, totalling 25 minutes per subject). A cough monitoring algorithm was applied, and the tool validated against cough counting by the developers. The device was reported to have a specificity of $94 \%$ and sensitivity of $96 \%$. However, during stair climbing specificity dropped to $87 \%$ with a sensitivity of $97 \%$. Correlation with manual counts was strong, $\mathrm{r}=0.94(86)$.

However, subsequent independent assessment of the Pulmotrack-CC ${ }^{\mathrm{TM}}$ was undertaken using recordings lasting up to 20 hours from 10 patients with chronic cough due to different chronic respiratory conditions. In this context the system was demonstrated to have poor agreement with cough counting by a human investigator, with a sensitivity of only $26 \%$ compared to coughs identified by ear (28). Until this lack of consistency is addressed the system cannot be reliably used.

\section{The Hull Automatic Cough Counter (HACC)}

The HACC records sound data from a wearable microphone over 24 hours. The signal is analysed to identify sound, and periods of silence are then omitted. The HACC was developed in 33 subjects with chronic cough, 23 of whom contributed data to determine reference cough features, with the remaining 10 subjects used for validation. The HACC was able to significantly reduce counting time compared to manual systems, taking approximately 1 minute 35 seconds to provide a count on 1 hour of data. However, the false positive rate of the automated system was high at almost $20 \%$ (sensitivity $80 \%$ and specificity $96 \%$ ) due to inability of the system to distinguish surrounding coughs from those produced by the subject (53). Comparing the HACC to manual counts in 10 subjects demonstrated strong correlation $(\mathrm{r}=0.87, \mathrm{P} \leq 0.001)$, but the HACC consistently did not count around a quarter of cough sounds identified by manual counting alone (87). As a result, it has not been adopted.

\section{Cayetano Cough Monitor}

The Cayetano Cough Monitor is a semi-automated system comprising a digital recording device and freefield microphone, recently developed by a Peru-based group studying TB (60). The device is reported to have a sensitivity of $75.5 \%$ in the ambulatory setting, with a false positive result of 4 events/hour. The operator must review approximately $5 \%$ of the total recording time for this sensitivity to be achieved (60). Unlike the other monitoring systems, the developers chose to count coughing bouts, as 
described above, rather than individual cough sounds which will make comparison with cough frequency measured by other devices difficult. This counting method was chosen as the monitor was found to have a sensitivity of only $51.4 \%$ when counting individual cough sounds. The device was used to determine cough frequency in pulmonary TB; 97 adults were enrolled in the study, who contributed 957 recordings. However, 685 of 1,642 (42\%) recordings were excluded due to high levels of background noise. The researchers are working to resolve this technical limitation by incorporating accelerometer-based technology (21).

\section{$L C M$}

In 2008, Birring et al. overcame previous limitations of 24-hour cough recording by developing and validating the LCM. The LCM is a small, lightweight system comprising a commercially-available portable digital recording device and free-field lapel microphone. The recorded data is subsequently analysed using an automated algorithm capable of detecting most cough sounds whilst rejecting noncough noises $(30,65)$. Operator input is required only for calibration as a consistency check to improve the specificity of the device, and takes approximately 5 minutes for every 24-hour recording. Validation was initially undertaken with data collected from 15 patients with chronic cough and 8 healthy volunteers. Reported sensitivity and specificity were $91 \%$ and $99 \%$, respectively for identification of cough sounds, and a median false positive rate of 2.5 events/patient/hour (30). The LCM was also shown to be repeatable over $>3$ months, and demonstrated a marginal improvement in repeatability when compared to manually analysed recordings (30).

Further evaluation by an independent researcher from another institution compared non-automated cough counts in $24 \mathrm{~h}$ recordings from 7 patients with idiopathic chronic cough to analysis by the machine. Automated and nonautomated cough counts were very similar (mean $\pm \mathrm{SE}$ : $23 \pm 7$, compared to $24 \pm 6$ coughs/patient/h respectively; intra-class correlation coefficient 0.98$)$ (88).

More recent testing of the LCM in 24-hour recordings from 20 individuals ( 8 healthy volunteers and 12 with chronic cough) showed a sensitivity of the system of $83.8 \%$ in patients and $82.3 \%$ in healthy volunteers, with a specificity of $99.9 \%$ in comparison to counting by ear (89).

The LCM has been used to obtain outcome measure data of cough frequency in a number of studies, including randomised controlled trials of gabapentin (90) and erythromycin (91) in chronic cough, and inhaled sodium cromoglycate in idiopathic pulmonary fibrosis (92). The system also been used successfully for measuring cough frequency in bronchiectasis (93), sarcoidosis (94), COPD (95), and TB (22).

\section{VitalofAK ${ }^{\mathrm{TM}}$}

The VitaloJAK ${ }^{\mathrm{TM}}$ was developed through collaboration between Vitalograph (a medical diagnostic device company) and the University Hospital of South Manchester. The system uses a combination of a lapel microphone, and contact microphone attached to the upper sternum with a specially-designed ambulatory recording device worn in a belt bag. Rather than generating cough counts by an automated process, the VitaloJAK ${ }^{\mathrm{TM}}$ software algorithm subsequently compresses audio recordings by removing all silent periods and the majority of non-cough sounds. Experienced operators then listen to the compressed recordings, each lasting approximately 1.5 hours per 24-hour monitoring period. Coughs are detected using an audio-visual display.

There have so far been two published reports on the evaluation of the software algorithm in a total of 30 individuals comprising 24 patients (with chronic cough, asthma or COPD) and 6 healthy controls $(96,97)$. The developers report an almost zero error rate in transferring the original cough sounds to the condensed recording apart from in one patient with asthma and apparently muffled cough sounds (96). The VitaloJAK ${ }^{\mathrm{TM}}$ cough frequency detection system has not undergone separate independent evaluation.

The VitaloJAK ${ }^{\mathrm{TM}}$ has been used to detect cough counts in a number of studies in a range of diseases including chronic cough, COPD, pulmonary fibrosis, asthma and cystic fibrosis (98-102). It has also successfully provided positive primary outcome efficacy data in Phase 2 studies of gefapixant in unexplained chronic cough $(19,20)$.

\section{Comparing the LCM and the VitalofAK ${ }^{\mathrm{TM}}$}

The LCM and VitaloJAK ${ }^{\mathrm{TM}}$ are the two most widely-used cough monitoring systems to date. They have not been directly compared, but lead to very similar 24-hour cough counts in similar types of patients [e.g., with chronic cough $(89,103)$, and acute cough $(104,105)]$. Both systems can record data continuously for 24 hours, with the LCM capable of doing so for up to 4 days (106). The LCM 
requires significantly less operator time. Conversely VitaloJAK ${ }^{\mathrm{TM}}$, mainly due to the greater human operator may have greater accuracy, in terms of correctly detecting all cough events, but this is difficult to quantify from published data. The VitaloJAK ${ }^{\text {TM }}$ has been used in children (107) and is safety tested as a medical device, although the LCM too could probably undergo the necessary adaptation and validation process to demonstrate its use in children.

The VitaloJAK ${ }^{\mathrm{TM}}$ recording system was designed specifically for cough sounds, whereas the audio capture equipment of the LCM was developed primarily for recording speech. However, as the LCM employs a microphone of high frequency response, and digitally records sound with sampling rate appropriate to cough sounds it is unclear if the bespoke recorder offers any advantage (30). The LCM recording is smaller and lighter, making it potentially more practical and acceptable to the wearer.

The lack of a chest wall contact microphone might make the LCM algorithm potentially more likely to overestimate cough counts if other individuals in the vicinity are also coughing. However, contact microphones have the disadvantage of being highly sensitive to noise from movement artefact, as previously discussed (41). Furthermore, due to the characteristics of the free-field microphone used, and the fact that distant-sounding coughs can be filtered out by the operator during the calibration phase of audio analysis, the accidental detection of background coughs might not be a significant problem in clinical use (108). Kulnik et al. tested the LCM on a hospital ward in which the wearer of the device and others in the background were prompted to cough. An observer remained present and counted coughs from both the study subject and others in the background in real time. Agreement between cough counts of the subjects from the LCM and the observer was extremely high [proportion of exact agreements 0.92; kappa 0.84, 95\% CI: 0.75, 0.93; device specificity $91 \%$ (95\% CI: $0.82,0.96)$ for detecting the study subject's cough only] (108).

The lack of direct skin contact with the LCM might make the monitor less noticeable by the wearer, potentially important if trying to determine 'usual' cough frequency. Conversely, as the LCM is easier to remove and replace, the wearer might potentially be more tempted to do this during a recording period. This might be easily more recognised with the VitaloJAK ${ }^{\mathrm{TM}}$ system as a lack of recorded data from the contact microphone, and the greater human input into analysis of recordings.

The relatively low unit cost of the LCM recording equipment allows it better feasibility for recordings in the home environment. In particular this facilitates sending the equipment by mail at the end of a recording period, rather than delivering it in person, increasing convenience for both study subjects and researchers. The low cost of the LCM hardware has also made it well-suited for independently-led collaborative non-commercial research, including in TB in East London and South Africa $(22,109)$.

The two systems therefore have different strengths and weaknesses and should probably be seen as complementary, both having supported significant recent advances in cough research (Table 1).

\section{Future directions}

At the present time cough monitoring very much remains a niche research tool. No well-validated automated cough frequency system is currently freely or commercially available. Both the LCM and the VitaloJAK ${ }^{\mathrm{TM}}$ systems offer substantial advances on the only previous alternatives for cough counting, but as discussed they have limitations, and are currently only accessible through research collaboration with the developers. Given the advances in the last two decades in computer and mobile technology, including in particular in speech recognition, developing a cough monitor with the ideal characteristics mentioned above should not seem beyond expectations. However, there has been very little financial investment in cough counting tools to date.

Despite the ease with which the human ear recognises coughs (presumably for reasons which have been favoured by natural selection), automated cough detection remains a challenge. As previously discussed, the approaches taken to automatic speech recognition are relevant, but owing to the noise-like qualities of cough compared to speech sounds, are only part of the solution. Further research focus is needed. Algorithms should be personalised to adapt to the cough sounds of the subject under observation in order that the coughs of others in the vicinity are ignored.

Future systems should also ideally operate in real time, recognising coughs as soon as they occur and ignoring all other sounds. In this way capturing sound information over the entirety of the recording period would become redundant. The currently-used cough monitors discussed above have this requirement, creating large digital data files for analysis at a later time point. Such an advance would 
Table 1 Ambulatory automatic and semi-automatic cough counting tools

\begin{tabular}{|c|c|c|c|c|c|}
\hline Device & Device & Components & Automation & Reported accuracy & Comments \\
\hline LR102 (85) & $\begin{array}{l}\text { Custom-built } \\
\text { device }\end{array}$ & $\begin{array}{l}3 \text { EMG sensors and } \\
\text { a contact sound } \\
\text { transducer }\end{array}$ & Full & $\begin{array}{l}\text { ICC, } r=0.87 \text { for number of } \\
\text { cough episodes/hour and } \\
r=0.89 \text { for number of single } \\
\text { coughs/hour compared to } \\
\text { manual counting of video } \\
\text { recording }\end{array}$ & $\begin{array}{l}\text { Overestimates cough frequency, } \\
\text { mean difference between the } \\
\text { meter and manual counts was } \\
3.8 \text { for cough episodes per hour } \\
(P=0.04) \text { and } 12.5 \text { for single } \\
\text { coughs per hour }(P<0.01)\end{array}$ \\
\hline $\begin{array}{l}\text { Pulmotrack- } \\
\mathrm{CC}^{\mathrm{TM}}(86)\end{array}$ & $\begin{array}{l}\text { Custom-built } \\
\text { device }\end{array}$ & $\begin{array}{l}\text { Two contact } \\
\text { microphones and a } \\
\text { pneumogram belt }\end{array}$ & Full & $\begin{array}{l}\text { Sensitivity of } 96 \% \text {; } \\
\text { specificity of } 94 \%\end{array}$ & $\begin{array}{l}\text { Independent validation reported } \\
\text { sensitivity of } 26 \% \text { compared to } \\
\text { coughs identified by ear ( } 28)\end{array}$ \\
\hline $\begin{array}{l}\text { Cayetano Cough } \\
\text { Monitor (60) }\end{array}$ & $\begin{array}{l}\text { MP3 digital } \\
\text { recorder }\end{array}$ & $\begin{array}{l}\text { Free-field } \\
\text { microphone }\end{array}$ & Partial & $\begin{array}{l}\text { Sensitivity } 96 \% \text {; specificity } \\
94 \% \text {; but reduces to } 75.5 \% \\
\text { in the ambulatory setting, } \\
\text { with a false positive result } \\
\text { of } 4 \text { events/hour }\end{array}$ & $\begin{array}{l}\text { Measures bouts rather than } \\
\text { sounds. Sensitivity of only } \\
51.4 \% \text { when counting individual } \\
\text { cough sounds }\end{array}$ \\
\hline $\begin{array}{l}\text { Leicester Cough } \\
\text { Monitor (30) }\end{array}$ & $\begin{array}{l}\text { MP3 digital } \\
\text { recorder }\end{array}$ & $\begin{array}{l}\text { Free-field } \\
\text { microphone }\end{array}$ & Partial & $\begin{array}{l}\text { Sensitivity 91\%; } \\
\text { specificity } 99 \%\end{array}$ & $\begin{array}{l}\text { False positive rate } 2.5 \text { events/ } \\
\text { patient/hour and repeatable } \\
\text { over } \geq 3 \text { months. Has been used } \\
\text { in commercial antitussive trials }\end{array}$ \\
\hline
\end{tabular}

not only have the advantage of simplifying data storage, but would be potentially very attractive to the individual being monitored. Current systems record not only coughs, but also speech and other environmental sounds, the nature and content of which may be personal and confidential. Research participants undergoing cough monitoring are made aware of this fact, and it may be a barrier to study recruitment. Capturing only coughs and ignoring other sounds should increase the acceptability of cough monitoring to individuals and regulators alike, and help lead to the wider use of cough monitoring in general.

Ongoing developments in cough counting tools, and the generation of more data, should go hand in hand with the wider application of cough frequency measurement, which in turn should lead to further advances in technology. Basic questions remain poorly answered in cough research, regarding, for example, the epidemiology of chronic cough in the general population, the normal range of cough frequency in health, and the extent to which cough counts vary from day to day within individuals in health and in stable respiratory disease. These points are key to better definitions of disease, for measuring responses to treatments, and for powering clinical trials, and should be addressed as cough frequency monitoring becomes more commonplace.

\section{Duration of cough monitoring}

The optimum duration for measuring cough frequency is not defined, but will probably relate in part to the question being asked. Clinical trials of new antitussives have recently used both 24-hour and daytime cough frequency $(19,110)$. 
It is not clear which is superior. It has been suggested that continuous monitoring for periods of only a few hours might be sufficient surrogate to assess daily cough frequency (111), but more data are needed. As technology now allows, recording periods of $48 \mathrm{~h}$ or longer may be preferred. This would not only allow more account to be taken of normal diurnal and inter-day variations in cough frequency, but also of any effect on cough frequency of wearing a monitoring device.

This theoretical effect has long been recognised but has not been quantified; cough monitoring may alter the subject's awareness of their cough, leading to fewer or more voluntary coughs, or avoiding activities which produce more coughing such as smoking $(32,112)$. Wearing a cough monitor over longer periods than $24 \mathrm{~h}$ might allow the subject to become accustomed to the device and not adapt behaviour as a result.

\section{Novel applications}

In a proof-of-concept study, Crooks et al. have demonstrated declines in cough frequency during recovery from exacerbations of COPD. Serial recordings were made with the LCM over several weeks in the home environment (113). Cough monitoring could potentially be similarly used to detect the early phases of COPD exacerbations, leading to more prompt interventions to mitigate against deterioration. This approach is also being investigated in asthma $(61,114)$, and could combine well with telemedicine for remote monitoring by health teams (115).

Cough frequency measurement could be a novel objective marker of disease severity, and of directly monitoring response to treatment. This has been investigated in TB $(21,116)$, a disease in which the currently-used objective markers of treatment response, including weight gain, radiographic improvement, and sterilisation of sputum microscopy are limited in responsiveness, sensitivity and specificity $(117,118)$. Cough monitoring could also impact on the management of interstitial lung diseases, also in need of better clinical markers of severity and treatment response (119).

Cough frequency has been investigated in the 1960s as a marker of infectiousness in TB (120), but only recently been shown to be feasible and potentially valuable using modern technology (22). Improved identification of the most infectious individuals would have clear advantages to the control of disease transmission (121). Screening for disease in apparently healthy individuals, particularly in its early stages has attracted a lot of attention, particularly in lung cancer $(122,123)$ and TB (124), and also has clear applications for the control of other respiratory infections (125). Cough counting tools could lend themselves well to screening, cough being a common and early feature of respiratory conditions. Cough monitoring would clearly first have to become much simplified and more widespread.

\section{Mobile device technology}

In early 2020 approximately $45 \%$ of the world's population owned a smartphone, up to $>90 \%$ in some parts of the world (126). Such devices commonly incorporate microphones, software and processors of sufficient specification to support accurate speech recognition systems. The potential adaptation of mobile devices as cough monitors therefore is very attractive.

One such smartphone application is currently in development and records, encrypts and transmits data to a remote, secure cloud server for automated analysis. This system awaits proper validation (127) and others are development (71). The continuous transmission of data for analysis remotely would circumvent the need to embed further signal processing capability for real-time cough detection within mobile phones themselves. However, the computational cost of running complex processing and classification algorithms, and the challenge of low signal-tonoise ratio for real-time cough detection could potentially be overcome with advances and adaptations in machine learning algorithms as discussed (71).

Smart electronic speakers are rapidly becoming more present in the home environment, and could also potentially adapted to monitor cough (128). The adaptation of commonly-owned electronic devices to monitor health is already proven, for example in screening for atrial fibrillation with a wristwatch (129), and should make cough counting technology more widely available, practical and acceptable to individuals.

\section{Conclusions}

We predict that the recent increased interest in the field of cough, accompanied by significant potential breakthroughs in new antitussive treatments, and rapid advances in mobile technologies and signal processing will lead to improvements and the much wider use of cough counting tools. Machines may ultimately surpass the human ear's ability to detect cough, perhaps removing any operator 
dependence of the systems.

With the increasing automation of modern life and reliance on technology such devices should have appeal not only to clinicians but also to patients alike, allowing them increased facility to monitor and manage their own condition. For healthcare services, they may have the added benefit of providing a remote method for tracking patient cohorts, and for screening for disease.

\section{Acknowledgments}

Funding: None.

\section{Footnote}

Provenance and Peer Review: This article was commissioned by the Guest Editor (Kefang Lai) for the series "3rd International Cough Conference" published in fournal of Thoracic Disease. The article was sent for external peer review.

Conflicts of Interest: All authors have completed the ICMJE uniform disclosure form (available at http:// dx.doi.org/10.21037/jtd-2020-icc-003). The series "3rd International Cough Conference" was commissioned by the editorial office without any funding or sponsorship. Dr. SB reports other from Avalyn, other from Patara, outside the submitted work; and Dr. SB is developer of LCM. The other authors have no other conflicts of interest to declare.

Ethical Statement: The authors are accountable for all aspects of the work in ensuring that questions related to the accuracy or integrity of any part of the work are appropriately investigated and resolved.

Open Access Statement: This is an Open Access article distributed in accordance with the Creative Commons Attribution-NonCommercial-NoDerivs 4.0 International License (CC BY-NC-ND 4.0), which permits the noncommercial replication and distribution of the article with the strict proviso that no changes or edits are made and the original work is properly cited (including links to both the formal publication through the relevant DOI and the license). See: https://creativecommons.org/licenses/by-nc-nd/4.0/.

\section{References}

1. Canning BJ, Chang AB, Bolser DC, et al. Anatomy and
Neurophysiology of Cough. Chest 2014;146:1633-48.

2. Cho PSP, Birring SS, Fletcher H, et al. Methods of Cough Assessment. J Allergy Clin Immunol Pract 2019;7:1715-23.

3. Leconte S, Ferrant D, Dory V, et al. Validated Methods of Cough Assessment: A Systematic Review of the Literature. Respiration 2011;81:161-74.

4. Spinou A, Birring SS. An update on measurement and monitoring of cough: What are the important study endpoints? J Thorac Dis 2014;6:S728-34.

5. Boulet LP, Coeytaux RR, McCrory DC, et al. Tools for assessing outcomes in studies of chronic cough: CHEST guideline and expert panel report. Chest 2015;147:804-14.

6. Morice AH, Fontana GA, Belvisi MG, et al. ERS guidelines on the assessment of cough. Eur Respir J 2007;29:1256-76.

7. Birring SS, Prudon B, Carr A, et al. Development of a symptom specific health status measure for patients with chronic cough: Leicester Cough Questionnaire (LCQ). Thorax 2003;58:339-43.

8. Birring SS, Parker D, Brightling CE, et al. Induced Sputum Inflammatory Mediator Concentrations in Chronic Cough. Am J Respir Crit Care Med 2004;169:15-9.

9. Vernon M, Kline Leidy N, Nacson A, et al. Measuring cough severity: development and pilot testing of a new seven-item cough severity patient-reported outcome measure. Ther Adv Respir Dis 2010;4:199-208.

10. French CT, Irwin RS, Fletcher KE, et al. Evaluation of a cough-specific quality-of-life questionnaire. Chest 2002;121:1123-31.

11. Brignall K, Jayaraman B, Birring SS. Quality of life and psychosocial aspects of cough. In: Lung. Springer; 2008;186:S55-8.

12. Birring SS, Matos S, Patel RB, et al. Cough frequency, cough sensitivity and health status in patients with chronic cough. Respir Med 2006;100:1105-9.

13. Lee KK, Matos S, Ward K, et al. Sound: A non-invasive measure of cough intensity. BMJ Open Respir Res 2017;4:e000178.

14. Kulnik ST, Birring SS, Hodsoll J, et al. Higher cough flow is associated with lower risk of pneumonia in acute stroke. Thorax 2016;71:474-5.

15. Earis J, Smith J. Analysis of the cough sound. In: Redington AE, Morice AH, editors. Acute and Chronic Cough. Boca Raton, Florida: Taylor \& Francis Group; 2005:143-60.

16. Song WJ, Chang YS, Faruqi S, et al. The global 
epidemiology of chronic cough in adults: a systematic review and meta-analysis. Eur Respir J 2015;45:1479-81.

17. Chamberlain SA, Garrod R, Douiri A, et al. The impact of Chronic cough: a cross sectional European survey. Lung 2015;193:401-8.

18. McGarvey LP, Carton C, Gamble L, et al. Prevalence of psychomorbidity among patients with chronic cough. Cough 2006;2:4.

19. Abdulqawi R, Dockry R, Holt K, et al. P2X3 receptor antagonist (AF-219) in refractory chronic cough: A randomised, double-blind, placebo-controlled phase 2 study. Lancet 2015;385:1198-205.

20. Smith JA, Kitt MM, Morice AH, et al. Gefapixant, a P2X3 receptor antagonist, for the treatment of refractory or unexplained chronic cough: a randomised, double-blind, controlled, parallel-group, phase $2 \mathrm{~b}$ trial. Lancet Respir Med 2020;8:775-85.

21. Proaño A, Bravard MA, López JW, et al. Dynamics of Cough Frequency in Adults Undergoing Treatment for Pulmonary Tuberculosis. Clin Infect Dis 2017;64:1174-81.

22. Turner RD, Birring SS, Darmalingam M, et al. Daily cough frequency in tuberculosis and association with household infection. Int J Tuberc Lung Dis 2018;22:863-70.

23. Windmon A, Minakshi M, Bhart P et al. TussisWatch: A Smart-Phone System to Identify Cough Episodes as Early Symptoms of Chronic Obstructive Pulmonary Disease and Congestive Heart Failure. IEEE J Biomed Health Inform 2019;23:1566-73.

24. Fontana GA, Widdicombe J. What is cough and what should be measured? Pulm Pharmacol Ther 2007;20:307.

25. Morice AH. Rebuttal: cough is an expiratory sound. Lung 2008;186:S7-9.

26. Decalmer SC, Webster D, Kelsall AA, et al. Chronic cough: how do cough reflex sensitivity and subjective assessments correlate with objective cough counts during ambulatory monitoring? Thorax 2007;62:329-34.

27. Kelsall A, Decalmer S, Webster D, et al. How to quantify coughing: Correlations with quality of life in chronic cough. Eur Respir J 2008;32:175-9.

28. Turner RD, Bothamley GH. How to count coughs? Counting by ear, the effect of visual data and the evaluation of an automated cough monitor. Respir Med 2014;108:1808-15.

29. Kelsall A, Houghton LA, Jones H, et al. A novel approach to studying the relationship between subjective and objective measures of cough. Chest 2011;139:569-75.

30. Birring SS, Fleming T, Matos S, et al. The Leicester
Cough Monitor: preliminary validation of an automated cough detection system in chronic cough. Eur Respir J 2008;31:1013-8.

31. Gravenstein JS, Devloo RA, Beecher HK. Effect of antitussive agents on experimental and pathological cough in man. J Appl Physiol 1954;7:119-39.

32. Woolf CR, Rosenberg A. Objective assessment of cough suppressants under clinical conditions using a tape recorder system. Thorax 1964;19:125-30.

33. Loudon RG, Brown LC. Cough frequency in patients with respiratory disease. Am Rev Respir Dis 1967;96:1137-43.

34. Loudon RG, Romans WE. Cough-monitoring equipment. Med Res Eng 1967;6:25-7.

35. Salmi T, Sovijarvi ARA, Brander P, et al. Long-term recording and automatic analysis of cough using filtered acoustic signals and movements on static charge sensitive bed. Chest 1988;94:970-5.

36. Chang AB, Newman R, Phelan P, et al. A new use for an old Holter monitor: an ambulatory cough meter. Eur Respir J 1997;10:1637-9.

37. Smith J, Woodcock A. New Developments in the Objective Assessment of Cough. Lung 2008;186:S48-54.

38. Matthys H, Bleicher B, Bleicher U. Dextromethorphan and codeine: objective assessment of antitussive activity in patients with chronic cough. J Int Med Res 1983;11:92-100

39. Pounsford JC, Birch MJ, Saunders KB. Effect of bronchodilators on the cough response to inhaled citric acid in normal and asthmatic subjects. Thorax 1985;40:662-7.

40. Cox ID, Wallis P, Apps M, et al. An electromyographic method of objectively assessing cough intensity and use of the method to assess effects of codeine on the dose-response curve to citric acid. Br J Clin Pharmacol 1984;18:377-82.

41. Drugman T, Urbain J, Bauwens N, et al. Objective study of sensor relevance for automatic cough detection. IEEE J Biomed Health Inform 2013;17:699-707.

42. McGuinness K, Ward K, Reilly CC, et al. Muscle activation and sound during voluntary single coughs and cough peals in healthy volunteers: Insights into cough intensity. Respir Physiol Neurobiol 2018;257:42-50.

43. Hadjileontiadis LJ. Lung sounds: An advanced signal processing perspective. Synthesis Lectures on Biomedical Engineering. Morgan \& Claypool Publishers; 2008; Vol. 9.

44. Rao A, Huynh E, Royston TJ, et al. Acoustic Methods for Pulmonary Diagnosis. IEEE Rev Biomed Eng 
2019;12:221-39.

45. Drugman T, Urbain J, Bauwens N, et al. Audio and Contact Microphones for Cough Detection. In: Annual Conference of the International Speech Communication Association, INTERSPEECH. Portland (OR); 2012 Sep 9-13. Available online: http://citeseerx.ist.psu.edu/ viewdoc/download?doi=10.1.1.500.805\&rep=rep $1 \&$ type $=$ pdf

46. Moradshahi P, Chatrzarrin H, Goubran R. Cough sound discrimination in noisy environments using microphone array. In: IEEE Instrum Meas Technol Conf. Minneapolis (MN); 2013 May 6-9. Available online: https://ieeexplore. ieee.org/document/6555454

47. Moradshahi P, Chatrzarrin H, Goubran R. Improving the performance of cough sound discriminator in reverberant environments using microphone array. IEEE Internation Instrumentation and Measurement Technology Conference Proceeding. Graz (Austria); 2012 May 13-16. Available online: https://ieeexplore.ieee.org/ document/6229523

48. Olia PM, Sestini P, Vagliasindi M. Acoustic parameters of voluntary cough in healthy non-smoking subjects. Respirology 2000;5:271-5.

49. Murata A, Taniguchi Y, Hashimoto Y, et al. Discrimination of Productive and Non-Productive Cough by Sound Analysis. Intern Med 1998;37:732-5.

50. Adhi Pramono RX, Anas Imtiaz S, Rodriguez-Villegas E. Automatic Identification of Cough Events from Acoustic Signals. In: 41st Annual International Conference of the IEEE Engineering in Medicine and Biology Society (EMBC). Berlin (Germany); 2019 July 23-27. Available online: https://ieeexplore.ieee.org/document/8856420

51. Proaño A, Bravard MA, Tracey BH, et al. Protocol for studying cough frequency in people with pulmonary tuberculosis. BMJ Open 2016;6:e010365.

52. Oppenheim A V, Schafer RW. Discrete-Time Signal Processing. Third edition. Pearson (UK); 2009.

53. Barry SJ, Dane AD, Morice AH, et al. The automatic recognition and counting of cough. Cough 2006;2:8.

54. Cohen-McFarlane M, Goubran R, Knoefel F. Comparison of Silence Removal Methods for the Identification of Audio Cough Events. In: 41st Annual International Conference of the IEEE Engineering in Medicine and Biology Society (EMBC). Berlin (Germany); 2019 July 23-27. Available online: https:// ieeexplore.ieee.org/document/8857889

55. Miranda IDS, Diacon AH, Niesler TR. A Comparative Study of Features for Acoustic Cough Detection Using
Deep Architectures. In: 41st Annual International Conference of the IEEE Engineering in Medicine and Biology Society (EMBC). Berlin (Germany); 2019 July 23-27. Available online: https://ieeexplore.ieee.org/ document/8856412

56. Amrulloh YA, Abeyratne UR, Swarnkar V, et al. Automatic cough segmentation from non-contact sound recordings in pediatric wards. Biomed Signal Process Control 2015;21:126-36.

57. Amrulloh Y, Abeyratne U, Swarnkar V, et al. Cough Sound Analysis for Pneumonia and Asthma Classification in Pediatric Population. In: 6th International Conference on Intelligent Systems, Modelling and Simulation. Kuala Lumpur (Maylasia); 2015 Feb 9-12. Available online: https://ieeexplore.ieee.org/document/7311223

58. Sun Z, Purohit A, Yang K. CoughLoc: Location-Aware Indoor Acoustic Sensing for Non-Intrusive Cough Detection. Int Work Emerg Mob Sens Technol Syst Appl 2011.

59. Swarnkar V, Abeyratne UR, Chang AB, et al. Automatic Identification of Wet and Dry Cough in Pediatric Patients with Respiratory Diseases. Ann Biomed Eng 2013;41:1016-28.

60. Larson S, Comina G, Gilman RH, et al. Validation of an Automated Cough Detection Algorithm for Tracking Recovery of Pulmonary Tuberculosis Patients. PLoS One 2012;7:e46229.

61. Sterling M, Rhee H, Bocko M. Automated Cough Assessment on a Mobile Platform. J Med Eng 2014;2014:951621.

62. Liu J-M, You M, Wang Z, et al. Cough event classification by pretrained deep neural network. BMC Med Inform Decis Mak 2015;15:S2.

63. You M, Liu Z, Chen C, et al. Cough detection by ensembling multiple frequency subband features. Biomed Signal Process Control 2017;33:132-40.

64. Abeyratne UR, Swarnkar V, Setyati A, et al. Cough sound analysis can rapidly diagnose childhood pneumonia. Ann Biomed Eng 2013;41:2448-62.

65. Matos S, Birring SS, Pavord ID, et al. Detection of cough signals in continuous audio recordings using hidden Markov models. IEEE Trans Biomed Eng 2006;53:1078-83.

66. Drugman T, Urbain J, Dutoit T. Assessment of audio features for automatic cough detection. In: 19th European Signal Processing Conference. Barcelona (Spain); 2011 Aug 29-Sep 2. Available online: https://ieeexplore.ieee. org/document/7074201 
67. Monge-Alvarez J, Hoyos-Barcelo C, Lesso P, et al. Robust Detection of Audio-Cough Events Using Local Hu Moments. IEEE J Biomed Health Inform 2019;23:184-96.

68. Martinek J. Distinction of cough from other sounds produced by daily activities in the upper airways. Bratisl Lek Listy 2011;112:120-4.

69. Abaza AA, Day JB, Reynolds JS, et al. Classification of voluntary cough sound and airflow patterns for detecting abnormal pulmonary function. Cough 2009;5:8.

70. Kosasih K, Abeyratne UR, Swarnkar V, et al. Wavelet Augmented Cough Analysis for Rapid Childhood Pneumonia Diagnosis. IEEE Trans Biomed Eng 2015;62:1185-94.

71. Hoyos-Barcelo C, Monge-Alvarez J, Zeeshan Shakir M, et al. Efficient k-NN Implementation for Real-Time Detection of Cough Events in Smartphones. IEEE J Biomed Health Inform 2018;22:1662-71.

72. Monge-Álvarez J, Hoyos-Barceló C, Dahal K, et al. Audiocough event detection based on moment theory. Appl Acoust 2018;135:124-35.

73. Mohammadi H, Samadani AA, Steele C, et al. Automatic discrimination between cough and non-cough accelerometry signal artefacts. Biomed Signal Process Control 2019;52:394-402.

74. Shin SH, Hashimoto T, Hatano S. Automatic Detection System for Cough Sounds as a Symptom of Abnormal Health Condition. IEEE Trans Inf Technol Biomed 2009; 13:486-93.

75. Amoh J, Odame K. Deep Neural Networks for Identifying Cough Sounds. IEEE Trans Biomed Circuits Syst 2016;10:1003-11.

76. Pramono RXA, Imtiaz SA, Rodriguez-Villegas E. A coughbased algorithm for automatic diagnosis of pertussis. PLoS One 2016;11:e0162128.

77. Adhi Pramono RX, Anas Imtiaz S, Rodriguez-Villegas E. Automatic Cough Detection in Acoustic Signal using Spectral Features. In: 41st Annual International Conference of the IEEE Engineering in Medicine and Biology Society (EMBC). Berlin (Germany); 2019 July 2327. Available online: https://ieeexplore.ieee.org/abstract/ document/8857792

78. Sharan RV, Abeyratne UR, Member S, et al. Cough Sound Recognition 2019;66:485-95.

79. Lee KK, Birring SS. Cough and sleep. Lung 2010;188:S91-4.

80. Pavesi L, Subburaj S, Porter-Shaw K. Application and validation of a computerized cough acquisition system for objective monitoring of acute cough: A meta-analysis.
Chest 2001;120:1121-8.

81. Coyle MA, Keenan DB, Henderson LS, et al. Evaluation of an ambulatory system for the quantification of cough frequency in patients with chronic obstructive pulmonary disease. Cough 2005;1:3.

82. Munyard P, Busst C, Logan-Sinclair R, et al. A new device for ambulatory cough recording. Pediatr Pulmonol 1994;18:178-86.

83. Hamutcu R, Francis J, Karakoc F, et al. Objective monitoring of cough in children with cystic fibrosis. Pediatr Pulmonol 2002;34:331-5.

84. Corrigan DL, Paton JY. Pilot study of objective cough monitoring in infants. Pediatr Pulmonol 2003;35:350-7.

85. Leconte $S$, Liistro $G$, Lebecque $P$, et al. The objective assessment of cough frequency: accuracy of the LR102 device. Cough 2011;7:11.

86. Vizel E, Yigla M, Goryachev Y, et al. Validation of an ambulatory cough detection and counting application using voluntary cough under different conditions. Cough 2010;6:3.

87. Faruqi S, Thompaon R, Wright C, et al. Quantifying chronic cough: Objective versus subjective measurements. Respirology 2011;16:314-20.

88. Birring SS, Mann VM, Matos S, et al. From the authors (response to: The Leicester Cough Monitor: a semiautomated, semi-validated cough detection system?). Eur Respir J 2008;32:528-9.

89. Yousaf N, Monteiro W, Matos S, et al. Cough frequency in health and disease. Eur Respir J 2013;41 241-3.

90. Ryan NM, Birring SS, Gibson PG. Gabapentin for refractory chronic cough: A randomised, double-blind, placebo-controlled trial. Lancet 2012;380:1583-9.

91. Yousaf N, Monteiro W, Parker D, et al. Long-term lowdose erythromycin in patients with unexplained chronic cough: A double-blind placebo controlled trial. Thorax 2010;65:1107-10.

92. Birring SS, Wijsenbeek MS, Agrawal S, et al. A novel formulation of inhaled sodium cromoglicate (PA101) in idiopathic pulmonary fibrosis and chronic cough: a randomised, double-blind, proof-of-concept, phase 2 trial. Lancet Respir Med 2017;5:806-15.

93. Spinou A, Lee KK, Sinha A, et al. The Objective Assessment of Cough Frequency in Bronchiectasis. Lung 2017;195:575-85.

94. Sinha A, Lee KK, Rafferty GF, et al. Predictors of objective cough frequency in pulmonary sarcoidosis. Eur Respir J 2016;47:1461-71.

95. Cho PSP, Fletcher H V, Patel IS, et al. Cough reflex 
sensitivity in exacerbations of chronic obstructive pulmonary disease. In: European Respiratory Journal. European Respiratory Society (ERS); 2018:PA4056.

96. Barton A, Gaydecki P, Holt K, et al. Data reduction for cough studies using distribution of audio frequency content. Cough 2012;8:12.

97. McGuinness K, Holt K, Dockry R, et al. Validation of the VitaloJAK 24 hour ambulatory cough monitor. Thorax 2012;67:A159.

98. Smith JA, Decalmer S, Kelsall A, et al. Acoustic coughreflux associations in chronic cough: potential triggers and mechanisms. Gastroenterology 2010;139:754-62.

99. Smith J, Owen E, Earis J, et al. Cough in COPD. Chest 2006;130:379-85

100. Key AL, Holt K, Hamilton A, et al. Objective cough frequency in idiopathic pulmonary fibrosis. Cough 2010;6:4.

101. Marsden PA, Satia I, Ibrahim B, et al. Objective Cough Frequency, Airway Inflammation, and Disease Control in Asthma. Chest 2016;149:1460-6.

102. Smith JA, Owen EC, Jones AM, et al. Objective measurement of cough during pulmonary exacerbations in adults with cystic fibrosis. Thorax 2006;61:425-9.

103. Kelsall A, Decalmer S, McGuinness K, et al. Sex differences and predictors of objective cough frequency in chronic cough. Thorax 2009;64:393-8.

104.Lee KK, Matos S, Evans DH, et al. A longitudinal assessment of acute cough. Am J Respir Crit Care Med 2013;187:991-7.

105.Sunger K, Powley W, Kelsall A, et al. Objective measurement of cough in otherwise healthy volunteers with acute cough. Eur Respir J 2013;41:277-84.

106. Mazhari R, Fraser H, Rowland S, et al. The Assessment of Cough Frequency: The Leicester Cough Monitor Experience. Lung 2014;192:3-4.

107.Deblej Elghamoudi D, Sumner H, McGuiness K, et al. P241 The feasibility and validity of objective cough monitoring in children using an adult cough detection system. Thorax 2015;70:A198.1-A198.

108. Kulnik ST, Williams NM, Kalra L, et al. Cough frequency monitors: Can they discriminate patient from environmental coughs? J Thorac Dis 2016;8:3152-9.

109. Williams CM, Abdulwhhab M, Birring SS, et al. Exhaled Mycobacterium tuberculosis output and detection of subclinical disease by face-mask sampling: prospective observational studies. Lancet Infect Dis 2020;20:607-17.

110. Khalid S, Murdoch R, Newlands A, et al. Transient receptor potential vanilloid 1 (TRPV1) antagonism in patients with refractory chronic cough: a double-blind randomized controlled trial. J Allergy Clin Immunol 2014;134:56-62.

111.Lee KK, Savani A, Matos S, et al. Four-hour cough frequency monitoring in chronic cough. Chest 2012;142:1237-43.

112.Loudon RG. Smoking and cough frequency. Am Rev Respir Dis 1976;114:1033-6.

113. Crooks MG, Hayman Y, Innes A, et al. Objective Measurement of Cough Frequency During COPD Exacerbation Convalescence. Lung 2016;194:117-20

114. Rhee H, Miner S, Sterling M, et al. The development of an automated device for asthma monitoring for adolescents: methodologic approach and user acceptability. JMIR mHealth uHealth 2014;2:e27.

115.Portnoy JM, Waller M, De Lurgio S, et al. Telemedicine is as effective as in-person visits for patients with asthma. Ann Allergy Asthma Immunol 2016;117:241-5.

116. Turner RD, Repossi AC, Matos S, et al. Cough prevalence and frequency in pulmonary tuberculosis. Thorax 2014;69:A43-4.

117.Horne DJ, Royce SE, Gooze L, et al. Sputum monitoring during tuberculosis treatment for predicting outcome: systematic review and meta-analysis. Lancet Infect Dis 2010;10:387-94.

118. Turner RD. Cough in pulmonary tuberculosis: Existing knowledge and general insights. Pulm Pharmacol Ther 2019;55:89-94.

119. Wells AU. Forced vital capacity as a primary end point in idiopathic pulmonary fibrosis treatment trials: making a silk purse from a sow's ear. Thorax 2013;68:309-10.

120.Loudon RG, Spohn SK. Cough frequency and infectivity in patients with pulmonary tuberculosis. Am Rev Respir Dis 1969;99:109-11.

121. Turner RD, Bothamley GH. Cough and the transmission of tuberculosis. J Infect Dis 2015;211:1367-72.

122. Athey VL, Suckling RJ, Tod AM, et al. Early diagnosis of lung cancer: evaluation of a community-based social marketing intervention. Thorax 2012;67:412-7.

123. de Koning HJ, Van Der Aalst CM, De Jong PA, et al. Reduced lung-cancer mortality with volume CT screening in a randomized trial. N Engl J Med 2020;382:503-13.

124. Yuen CM, Amanullah F, Dharmadhikari A, et al. Turning off the tap: Stopping tuberculosis transmission through active case-finding and prompt effective treatment. Lancet 2015;386:2334-43.

125. Hoehl S, Berger A, Kortenbusch M, et al. Evidence of SARS-CoV-2 Infection in Returning Travelers from 
Wuhan, China. N Engl J Med 2020;382:1278-80.

126. Smartphone users worldwide 2020 I Statista [Internet]. [cited $2020 \mathrm{Feb}$ 20]. Available online: https://www.statista. com/statistics/330695/number-of-smartphone-usersworldwide/

127. Kvapilova L, Boza V, Dubec P, J, et al. Continuous Sound Collection Using Smartphones and Machine Learning to Measure Cough. Digit Biomark 2019;3:166-75.

Cite this article as: Hall JI, Lozano M, Estrada-Petrocelli L, Birring S, Turner R. The present and future of cough counting tools. J Thorac Dis 2020;12(9):5207-5223. doi: 10.21037/jtd2020-icc-003
128. Dr Alexa wants you to cough up for medicine I News I The Times [Internet]. [cited 2020 Feb 20]. Available online: https://www.thetimes.co.uk/article/dr-alexa-wantsyou-to-cough-up-for-medicine-cfxqwsj8d

129.Perez MV, Mahaffey KW, Hedlin H, et al. Large-Scale Assessment of a Smartwatch to Identify Atrial Fibrillation. New Engl J Med 2019;381:1909-17. 